\title{
Deep Learning Analysis of Polar Maps from SPECT Myocardial Perfusion Imaging for Prediction of Coronary Artery Disease
}

\author{
Narges Zahiri \\ Shahid Sadoughi University of Medical Sciences and Health Services \\ Rhona Asgari \\ Medical University of Vienna Department of Ophthalmology and Optometry \\ Seid-Kazem Razavi-Ratki \\ Shahid Sadoughi University of Medical Sciences and Health Services \\ Ali-Asghar parach ( $\square$ aliparach@gmail.com ) \\ Shahid Sadoughi University of Medical Sciences and Health Services https://orcid.org/0000-0003-0787-9575
}

\section{Research Article}

Keywords: Coronary artery disease, Polar map images, SPECT, Deep learning, Convolutional neural networks

Posted Date: December 15th, 2021

DOI: https://doi.org/10.21203/rs.3.rs-1153347/v1

License: (c) (1) This work is licensed under a Creative Commons Attribution 4.0 International License. Read Full License 


\section{Abstract}

Purpose: This study aimed to investigate the diagnostic accuracy of deep convolutional neural networks for classifying the polar map images in Singlephoton emission computed tomography (SPECT) myocardial perfusion imaging (MPI) by considering the physician's diagnosis as reference.

Methods: 3318 images of stress and rest polar maps related to patients (67\% women and 33\% men) who underwent ${ }^{99 \mathrm{~m}} \mathrm{Tc}$-sestamibi MPI were collected. The images were manually labeled with normal and abnormal labels according to the doctor's diagnosis reports. The proposed deep learning model was trained using stress and rest polar maps and evaluated for prediction of obstructive disease in a stratified 5-fold cross-validation procedure.

Results: The mean values of accuracy, sensitivity, accuracy, specificity, f1 score, and the area under the roc curve were $0.7562,0.7856,0.5748,0.7434$, 0.6646 , and, 0.8450 , respectively over 5 folds using both stress and rest scans. The inclusion of rest perfusion maps significantly improved AUC of the deep learning model (AUC: $0.845 ; 95 \% \mathrm{Cl}: 0.832-0.857)$, compared with using stress polar maps only (AUC: $0.827 ; 95 \%$ Cl: $0.814-0.840) ; P<0.05$.

Conclusion: The results of the present work reveal the possible applications of deep learning for polar map images classification in SPECT MPI.

\section{Introduction}

Coronary artery disease (CAD) is a topic of pivotal importance in heart disease, accounts for one in every five deaths, and is commonly seen throughout the world[1]. As a direct consequence of this mortality, there have been increasing calls for imaging techniques to predict the presence of CAD disease.

Single-photon emission computed tomography (SPECT) myocardial perfusion imaging (MPI) is widely used throughout the world to assess coronary artery disease[2]. This technique can provide information about myocardial perfusion which is crucial to know the significance and extent of coronary artery stenosis as well as information regarding ventricular function[3]. The key idea in SPECT MPI is to image the patient's heart twice, during the rest and under stress, to identify areas of reduced radiopharmaceutical uptake[4].

Visual assessment is often chosen as the default approach for perfusion image interpretation; however, this one could be a time-demanding task. Moreover, such a method is notably dependent on the experience of the nuclear medicine expert or radiologist and suffers from intra-observer and inter-observer variability. Therefore, to increase the reproducibility of reports, quantitative analysis is typically integrated into visual evaluation[2, 4]. The main focus of developing technologies in nuclear cardiology is on the automation and use of artificial intelligence algorithms as a component of the interpretation process[2].

The term DL has been introduced in an attempt to develop a method to boost the performance of conventional artificial neural networks (ANNs) using deep architectures and more layers[3,5]. The convolutional neural network (CNN) is a class of deep neural networks that are mainly used in image analysis such as medical images due to its unique feature of maintaining local relationships in the image while reducing dimensions[6, 7]. Contrary to traditional machine learning that normally requires pre-determined image measurements, CNNs connect directly to image pixels to learn image statistics on their own, so that image processing is done naturally [6]. This is a robust new machine learning tool, with breakthrough applications in medical imaging issues such as disease detection and classification[5, 8].

Several studies have explored the use of CNNS for the prediction and classification of obstructive coronary artery disease in the nuclear cardiology field [4, 6, 9-15]. Spir et al. (2019) suggested a method for the classification of polar maps based on a graph-type CNN and compared the agreement with the human observer[4]. Bentacor et al. (2019) used deep learning based on a combination of raw and quantitative supine and upright stress polar maps to predict obstructive coronary artery disease [6]. In a study by Apostolopoulos et al. (2020), a dataset of attenuation-corrected (AC) and non-attenuationcorrected (NAC) stress and rest polar maps and clinical data were analyzed using a multi-input network consisting of inceptionv3 and a random forest [9]. In the present work, a CNN based on a combination of stress and rest polar maps is introduced to classify coronary artery disease.

This study aimed to investigate the diagnostic accuracy of deep convolutional neural networks for classifying the polar map images in myocardial perfusion imaging by considering the physician's diagnosis as a reference.

\section{Materials And Methods \\ 2.1 Study Population}

The study population comprised 3,318 patients ( $67 \%$ women and $33 \%$ men) referred for SPECT MPI from 2018 to 2020 , at the nuclear medicine center. The study was approved by the Ethical Committee of our Institution. Polar maps in stress and rest conditions were extracted from the SPECT scan in tiff format. The images of stress and rest were resized to $221 \times 217$ with 3 channels, to remove the unnecessary parts and reduce the computational cost. Sample polar map images as identified in the data set are shown in Fig. 1 and Fig. 2.

\subsection{Image Acquisition}

The stress SPECT images were acquired about 30 min after an injection of 20 mCi Technetium-99m (Tc-99m MIBI) following an exercise test or pharmacological stress. Tc- $99 \mathrm{~m}$ MIBI was injected after at least $85 \%$ of the age-predicted maximum heart rate was achieved. Rest imaging was carried out nearly $40 \mathrm{~min}$ after an injection of 15-20 mCi Tc-99m MIBI. Two SPECT gamma-cameras (Philips Forte and Genesis cameras) equipped with low-energy high-resolution collimators, were used for MPI imaging. The data were collected from 32 projections of 25-30 seconds in a 140 Kev photo-peak over a 180degree arc in a 64x64 matrix. The stress and rest SPECT MPI were performed using 180 degrees SPECT imaging, beginning from 45 degrees right anterior-

Page 2/12 
oblique and ending at 45 degrees left posterior-oblique. The patients were imaged in the supine position. The short axis and vertical and horizontal longaxis slices were reconstructed from the raw projection data by filtered back projection or iterative algorithms in the rest and stress SPECT MPI. No attenuation correction was performed. Polar maps were extracted from SPECT images.

\subsection{MPI Interpretation}

A nuclear medicine expert had been engaged retrospectively in the interpretation of MPI studies. Myocardial perfusion imaging reports were considered positive for CAD if they depicted a reversible tracer defect of any extent. If a perfusion defect in the SPECT images acquired after exercise was not seen in the rest images, this condition was described as ischemia. For example, if the result is "Stress-induced ischemia of the apical lateral, apical anterior and mid anterolateral wall", the patient is labeled as abnormal. If the result is "The study is negative for appreciable stress-induced ischemia", the patient is labeled as normal. Among 3318 subjects in this study, 2303 subjects were labeled as normal and 1015 subjects as abnormal.

\subsection{Deep Learning Model}

The overall process is schematically shown in Fig. 3. Our network includes five convolution blocks with an increasing number of filters in succession to extract image features. Each block consists of two convolution layers. Each layer is followed by an exponential linear unit (ELU) activation function and a batch-normalization (BN) layer for normalizing data. Then after each block, there is a max-pooling layer with stride 2 that maintains only the maximum value from a $2 \times 2$ input, to compensate for small image changes and distortions. These convolution layers are used for obtaining the main features of input images. The early blocks extract low-level features whereas the other succeeding blocks allow for the detection of higher-level features. The output of convolution layers is passed through a global Average pooling (GAP) layer to generate the feature vector. The feature vector then passes to the second part. This part includes two fully connected layers (arrays of neurons linked with each neuron in the preceding layer), followed by a dropout and a dense layer with one node and a sigmoid function to generate the output class. A summary of the model is given in Table 1.

\subsection{Implementation}

Our proposed deep learning model was implemented using Python programming language version 3.7.10 and Keras Application Programming Interface (API) version 2.4.3 for Tensorflow version 2.4.1. The experiments were run using the graphical processor units on Google Colaboratory. Our model used focal loss function and adam optimizer. A focal loss function addresses the class imbalance problem during training a model[16]. Two hyper-parameters, alpha, and gamma are applied to the Cross-Entropy loss function. Alpha gives more importance to the minority class (abnormal class) and handles the class imbalance problem while gamma gives more importance to misclassified examples and makes the model efficient to learn for hard (misclassified) examples. Suitable values for alpha and gamma were 0.75 and 0.2 , respectively. The initial learning rate, batch size, and the number of epochs was 0.001 , 16 , and 50 , respectively. The learning rate was reduced by a factor of 0.2 when no improvement was seen on validation loss for 10 epochs. The lower bound on the learning rate was 0.00001 .

\subsection{Data Augmentation}

To reduce over-fitting and enable more generalization, in each of the k rounds during the cross-validation method, the training parts were augmented before the training. We rotated the stress and rest polar maps simultaneously and partly (by a maximum of 45 degrees). Then the rotated polar maps were concatenated along the channel dimension. In such a way, the model ignored small spatial differences between the polar maps and focused on the color variations, irrespective of their relative position.

\subsection{Cross-validation}

The validation of the deep learning model was performed using a stratified 5-fold cross-validation procedure to proper training and reliable evaluation of the CNN and maximizing the use of data while avoiding model over-fitting and selection bias. The approach randomly splits the training set into 5 nonoverlapping groups of patients of roughly equal size where the first four groups are the training set, while the fifth part remained hidden from the model for testing. These groups are stratified to have a similar percentage of obstructive disease as the studied population. Before the training, the four training parts were augmented. This procedure was repeated for the left parts until every part was selected for the test set. Finally, the mean of the metrics over five folds provides an overall performance estimate of the deep learning model.

\subsection{Added Value of Rest Scans}

The added value of rest perfusion maps was assessed by evaluating the deep learning model performance trained including rest perfusion polar maps in addition to stress polar maps. The 2 methods (with and without rest maps) were evaluated into the same cross-validation procedure, using the same folds.

\subsection{Statistical Analysis}

The diagnostic performance of the deep learning model was evaluated using classification metrics including accuracy, precision, recall, f1score, specificity, and the area under the ROC curve (AUC). The added value of rest polar maps was evaluated using ROC analysis and pairwise comparisons of the area under the ROC curve according to the DeLong test[17]. McNemar's chi-square test was also used to assess the significance of changes between two deep learning methods (with and without rest polar maps). A 2-tailed p-value $<0.05$ was considered statistically significant. Statistical tests were performed in MedCalc software version 20.0.15.

\section{Results}

\subsection{Five-fold cross-validation results}


Table 2 provides the average accuracy, AUC, recall, precision, f1-Score, and specificity metrics over 5 folds along with the corresponding standard deviations for two deep learning methods (with and without rest polar maps). The results related to considering rest in addition to stress polar maps are also shown graphically in Fig. 4 as box plots.

\subsection{Learning curves}

The average accuracy and error learning curves for the training and test data set over 5 folds are presented in Fig. 5 . The learning curve is the performance of a learning model over experience or time. Reviewing the learning curves of the models during training can be used to diagnose learning problems, such as over-fitting and under- fitting, as well as whether the training and validation data sets are appropriate. As seen in Fig. 5 , the training and test error curves decrease and converge together. The training and test accuracy curves also increase and converge at the same time and the iteration stops before overfitting.

\subsection{Confusion matrix}

Table 3 presents the summarized confusion matrix on total data obtained from the sum of the matrices of all folds. Also, the normalized confusion matrix in Table 4 is created by dividing each element by the sum of the corresponding row elements, which represents the number of images in each class. The values in this matrix belong to one of the True Negative, True Positive, False Negative, and False Positive categories.

\subsection{ROC and Precision-recall curves}

The Receiver Operating Characteristic curve, also known as the ROC, displays the relationship between True Positive Rate and False Positive Rate for each threshold value. To numerically represent the ROC curve, the Area under the Curve can be calculated. Fig. 6 on the left part illustrates the ROC curve for each of the 5 folds, as well as the overall curve obtained from the prediction on all data, along with the AUC values. Fig. 6 on the right part shows the precisionrecall curve for each of the 5 folds, as well as the overall curve obtained from the prediction on all data, along with the AUC values.

\subsection{The Result of Adding Rest Scans}

According to the DeLong test, the inclusion of rest perfusion maps significantly improved the AUC of the deep learning model (AUC: 0.845 ; $95 \%$ Cl: $0.832-$ 0.857), compared with using stress polar maps only (AUC: $0.827 ; 95 \% \mathrm{Cl}: 0.814-0.840$ ); $\mathrm{P}<0.05$ shown in Fig. 7. McNemar's test also shows that the difference before and after adding rest maps is $-2.68 \%$ with $95 \% \mathrm{Cl}$ from $-4.09 \%$ to $-1.27 \%$, which is significant $(P=0.0002)$.

\section{Discussion}

This study aimed to assess the feasibility of SPECT MPI polar maps classification using deep learning methods. The deep learning model was evaluated using a stratified 5-fold cross-validation strategy. Taking expert diagnosis as a reference, well-established metrics, including accuracy, AUC, sensitivity, and specificity, were computed to evaluate the performance of the proposed deep learning model.

Visual assessment of SPECT heart perfusion images faces several challenges, including the lack of reproducibility due to the intra-observer and interobserver variability, dependency on nuclear medicine expert or radiologist experience, and increased time and cost of the interpretation process. Machine learning and Deep learning techniques exhibited promising potential for the detection and classification of coronary artery disease from SPECT MPI images compared with other approaches, including human observer diagnosis and quantitative analysis of perfusion defects [4, 6, 9-15, 18-21]. A comparison to the related studies is listed in Table $5 . \ln [4,6,9,10,12,15]$, the authors used CNNs with polar maps.

A previously proposed DL-based method by Betancur et al. combining raw and quantitative supine and upright stress polar maps and patient gender information to predict obstructive coronary artery disease led to a greater per-patient and per-vessel AUC than the combined total perfusion deficit (TPD). (Per-patient, 0.81 vs. 0.78; per-vessel, 0.77 vs. $0.73 ; \mathrm{P}<0.001$ ).Considering the same cut-off and specificity for combined TPD and deep learning, the sensitivity improved from $61.8 \%$ (TPD) to $65.6 \%$ (DL) per patient; $P<0.05[6]$.

In a work by Yukaotaki et al. (2021), a total of 3,578 patients with suspected CAD undergoing SPECT MPI and invasive coronary angiography from 9 centers were studied. The deep learning model calculates the probability of obstructive CAD from stress myocardial perfusion, wall motion, and wall thickness maps, as well as left ventricular volume, age, and sex. In a validation method with 10 folds, the area under the ROC curve for DL (AUC: 0.83 ; $95 \%$ $\mathrm{Cl}$ 0.82-0.85) was significantly higher than the quantification of perfusion defects (AUC: $0.78 ; 95 \% \mathrm{Cl}: 0.77-0.80$ ) and expert opinion (AUC: 0.71 ; $95 \% \mathrm{Cl}$ : 0.69-0.72]; $P<0.0001$ for both). Rest scans were not considered in the above-mentioned studies[15].

Spir et al. (2019) suggested a method for the classification of polar maps based on a graph-type convolutional neural network using a 946 labeled dataset and compared it with three other neural network methods. The proposed model agreed with the human observer in $89.3 \%$ of the rest test polar maps and $91.1 \%$ of the stress test polar maps. The localization was performed on the 17-part segmentation of the polar maps and reached $83.1 \%$ agreement with the human observer. Among all related works dealing with polar maps, the highest accuracy is related to this study and is equal to $0.91 \%[4]$.

In a study by Apostolopoulos et al. (2020), a collection of 566 patient samples was analyzed. The dataset includes a combination of attenuation-corrected (AC) and non-attenuation-corrected (NAC) stress and rest polar maps, clinical data, and coronary angiography results, the latter of which is considered as the ground truth. The four polar maps corresponding to each patient were concatenated into one image alongside one another. This research shows that an optimal strategy involves a hybrid multi-input network consisting of inceptionv3 and a random forest and data augmentations with small shifts in any direction. This method corresponds to the expert's accuracy, which is $79.15 \%$ for this specific data set [9]. 
The current study deals with a combination of stress and rest polar maps as model input for each patient while most previous studies have used only stress polar maps as input to the convolutional network. We demonstrated that deep learning utilizing a combination of stress and rest perfusion polar maps outperforms deep learning using only stress polar maps for the prediction of obstructive disease. These findings suggest that the rest polar maps provide important supplementary information. This could be because the model uses the difference between two polar maps to detect obstructive disease or ischemia. Although our dataset is different from the corresponding works, the proposed method offers a competitive performance compared to mentioned related studies in terms of accuracy, sensitivity, specificity, and the area under the ROC curve which corresponds to $0.7562 \%, 0.7856 \%, 0.7434 \%$, and, $0.8450 \%$, respectively. The results also reflect those of Apostolopoulos et al. (2020) who also found that data augmentation by rotation is an effective strategy to prevent over-fitting in classifying polar maps[10].

This work suffers from some limitations. First, the data augmentation method to overcome the data shortage is not strong enough to create real images. In addition, by rotating the polar maps, the location of the abnormal perfusion is attributed to another area in the myocardium. However, in this study, we examined the overall diagnostic effectiveness of this method (normal or abnormal) and not its ability to locate findings in specific coronary artery areas. Furthermore, this work is also limited by its consideration of only polar map images.

This study could further be refined by considering the clinical and functional data. Also, Coronary angiography information can be used as a ground truth. Moreover, by increasing the number of images, higher performance can be achieved and the model generalization can be improved.

\section{Conclusion}

Despite the above limitations, the results of the present work reveal the possible applications of deep learning for polar map images classification in SPECT myocardial perfusion imaging. These findings suggest that our approach can be a promising alternative to routine polar map analysis using normal databases to further support the clinical decision-making process by providing a second opinion.

\section{Declarations}

\section{Funding:}

The authors declare that no funds, grants, or other support were received during the preparation of this manuscript.

\section{Competing Interests:}

The authors have no relevant financial or non-finantial interests to disclose. All authors contributed to the study conception and design.

\section{Author Contributions:}

Material preparation, data collection and analysis were performed by Narges Zahiri, Rhona Asgari, Seid-Kazem Razavi-Ratki, and ali asghar parach. The first draft of the manuscript was written by Narges Zahiri and all authors commented on previous versions of the manuscript. All authors read and approved the final manuscript.

\section{Ethics approval:}

This study was conducted with the approval of the Ethics Committee of Shahid Sadoughi University of Medical Sciences under the Ethics code IR.SSU.MEDICINE.REC. 1399.113, with a registration date of 13/09/2020.

\section{Consent to participate:}

The nature of the study waives the requirement to obtain informed consent.

\section{Consent to publish:}

The authors affirm that human research participants provided informed consent for publication of the images in Figures 1 and 2 and their names or information is not obvious in the manuscript.

\section{References}

1. Seetharam K, Shresthra S, Mills JD, Sengupta PP (2019) Artificial intelligence in nuclear cardiology: adding value to prognostication. Current Cardiovascular Imaging Reports 12:1-6

2. Gomez J, Doukky R, Germano G, Slomka P (2018) New trends in quantitative nuclear cardiology methods. Current cardiovascular imaging reports 11:1-10

3. Eid M, Spearman JV, van Assen M, De Santis D, Sahbaee P, Landreth SP, et al. Machine learning and artificial intelligence in cardiovascular imaging. CT of the Heart: Springer; 2019. p. 893-907.

4. Spier N, Nekolla S, Rupprecht C, Mustafa M, Navab N, Baust M (2019) Classification of Polar Maps from Cardiac Perfusion Imaging with GraphConvolutional Neural Networks. Scientific Reports 9:7569.https://doi.org/10.1038/s41598-019-43951-8 
5. Currie G (2019) Intelligent imaging: anatomy of machine learning and deep learning. Journal of nuclear medicine technology 47:273-81

6. Betancur J, Hu LH, Commandeur F, Sharir T, Einstein AJ, Fish MB, et al. (2019) Deep Learning Analysis of Upright-Supine High-Efficiency SPECT Myocardial Perfusion Imaging for Prediction of Obstructive Coronary Artery Disease: A Multicenter Study. Journal of nuclear medicine : official publication, Society of Nuclear Medicine 60:664-70

7. Ker J, Wang L, Rao J, Lim T (2017) Deep learning applications in medical image analysis. leee Access 6:9375-89

8. Visvikis D, Le Rest CC, Jaouen V, Hatt M (2019) Artificial intelligence, machine (deep) learning and radio (geno) mics: definitions and nuclear medicine imaging applications. European journal of nuclear medicine and molecular imaging 46:2630-7

9. Apostolopoulos ID, Apostolopoulos DI, Spyridonidis TI, Papathanasiou ND, Panayiotakis GS (2021) Multi-input deep learning approach for Cardiovascular Disease diagnosis using Myocardial Perfusion Imaging and clinical data. Physica medica : PM : an international journal devoted to the applications of physics to medicine and biology : official journal of the Italian Association of Biomedical Physics (AIFB) 84:168-77

10. Apostolopoulos ID, Papathanasiou ND, Spyridonidis T, Apostolopoulos DJ (2020) Automatic characterization of myocardial perfusion imaging polar maps employing deep learning and data augmentation. Hellenic journal of nuclear medicine 23:125-32

11. Berkaya SK, Sivrikoz IA, Gunal S (2020) Classification models for SPECT myocardial perfusion imaging. Computers in Biology and Medicine 123:103893

12. Betancur J, Commandeur F, Motlagh M, Sharir T, Einstein AJ, Bokhari S, et al. (2018) Deep learning for prediction of obstructive disease from fast myocardial perfusion SPECT: a multicenter study. JACC: Cardiovascular Imaging 11:1654-63

13. Chen J-J, Su T-Y, Chen W-S, Chang Y-H, Lu HH-S (2021) Convolutional neural network in the evaluation of myocardial ischemia from czt spect myocardial perfusion imaging: Comparison to automated quantification. Applied Sciences 11:514

14. Liu H, Wu J, Miller EJ, Liu C, Liu Y-H (2021) Diagnostic accuracy of stress-only myocardial perfusion SPECT improved by deep learning. European Journal of Nuclear Medicine and Molecular Imaging 1-8

15. Otaki Y, Singh A, Kavanagh P, Miller RJH, Parekh T, Tamarappoo BK, et al. (2021) Clinical Deployment of Explainable Artificial Intelligence of SPECT for Diagnosis of Coronary Artery Disease. JACC: Cardiovascular

Imaging.https://www.sciencedirect.com/science/article/pii/S1936878X21004381.https://www.sciencedirect.com/science/article/pii/S1936878X21004381

16. Ross T-Y, Dollár G, editors. Focal loss for dense object detection. Proceedings of the IEEE Conference on Computer Vision and Pattern Recognition; 2017.

17. DeLong ER, DeLong DM, Clarke-Pearson DL (1988) Comparing the areas under two or more correlated receiver operating characteristic curves: a nonparametric approach. Biometrics 44:837-45

18. Betancur J, Otaki Y, Motwani M, Fish MB, Lemley M, Dey D, et al. (2018) Prognostic Value of Combined Clinical and\&\#xa0;Myocardial Perfusion Imaging Data Using Machine Learning. JACC: Cardiovascular Imaging 11:1000-9.https://www.jacc.org/doi/abs/10.1016/j.jcmg.2017.07.024

19. Nakajima K, Kudo T, Nakata T, Kiso K, Kasai T, Taniguchi Y, et al. (2017) Diagnostic accuracy of an artificial neural network compared with statistical quantitation of myocardial perfusion images: a Japanese multicenter study. European journal of nuclear medicine and molecular imaging 44:2280-9

20. Rahmani R, Niazi P, Naseri M, Neishabouri M, Farzanefar S, Eftekhari M, et al. (2019) Improved diagnostic accuracy for myocardial perfusion imaging using artificial neural networks on different input variables including clinical and quantification data. Revista Española de Medicina Nuclear e Imagen Molecular (English Edition) 38:275-9

21. Yoneyama H, Nakajima K, Taki J, Wakabayashi H, Matsuo S, Konishi T, et al. (2019) Ability of artificial intelligence to diagnose coronary artery stenosis using hybrid images of coronary computed tomography angiography and myocardial perfusion SPECT. European Journal of Hybrid Imaging 3:1-14

22. Betancur J, Commandeur F, Motlagh M, Sharir T, Einstein AJ, Bokhari S, et al. (2018) Deep Learning for Prediction of Obstructive Disease From Fast Myocardial Perfusion SPECT: A Multicenter Study. JACC Cardiovascular imaging 11:1654-63

\section{Tables}


Table 1: Summary of the suggested model

\begin{tabular}{|c|c|c|c|c|}
\hline & Layer Type & Filters & Output Shape & Kernel Size/Strides \\
\hline Input & Image(stress+rest) & 6 & $221 * 217 * 6$ & - \\
\hline Block-1 & 2* [Convolution+Elu+BN] & 32 & $213 * 209 * 32$ & $5 * 5 / 1$ \\
\hline- & Max-pooling & 32 & $106 * 104 * 32$ & $2 * 2 / 2$ \\
\hline Block-2 & $2 *[$ Convolution+Elu+BN] & 64 & $102 * 100 * 64$ & $3 * 3 / 1$ \\
\hline- & Max-pooling & 64 & $51 * 50 * 64$ & $2 * 2 / 2$ \\
\hline Block-3 & $2 *[$ Convolution + Elu $+\mathrm{BN}]$ & 64 & $47 * 46 * 64$ & $3 * 3 / 1$ \\
\hline- & Max-pooling & 64 & $23 * 23 * 64$ & $2 * 2 / 2$ \\
\hline Block-4 & $2 *[$ Convolution+Elu+BN] & 128 & $19 * 19 * 128$ & $3 * 3 / 1$ \\
\hline- & Max-pooling & 128 & $9 * 9 * 128$ & $2 * 2 / 2$ \\
\hline Block-5 & $2 *[$ Convolution+Elu+BN] & 128 & $5 * 5 * 128$ & $3 * 3 / 1$ \\
\hline- & Max-pooling & 128 & $2 * 2 * 128$ & $2 * 2 / 2$ \\
\hline- & GAP & - & 128 & $2 * 2 / 2$ \\
\hline- & Dense-1+Elu+BN & 64 & 64 & - \\
\hline- & Dense-2+Elu+BN & 32 & 32 & - \\
\hline- & Dropout(0.5) & 32 & 32 & - \\
\hline Output & Sigmoid & 1 & 1 & - \\
\hline
\end{tabular}

Table 2: Average Classification Metrics over 5 Folds

\begin{tabular}{ccccccc}
\hline & Accuracy & AUC & Recall & Precision & F1-score & Specificity \\
\hline $\begin{array}{c}\text { Average } \\
\text { (stress + rest) }\end{array}$ & $0.7562 \pm 0.0134$ & $0.8450 \pm 0.0135$ & $0.7856 \pm 0.0216$ & $0.5748 \pm 0.0191$ & $0.6646 \pm 0.0131$ & $0.7434 \pm 0.0223$ \\
\hline Average (stress) & $0.7209 \pm 0.0226$ & $0.8278 \pm 0.0148$ & $0.7714 \pm 0.0538$ & $0.5316 \pm 0.0272$ & $0.6283 \pm 0.0227$ & $0.6986 \pm 0.0425$ \\
\hline
\end{tabular}

Table 3: Total Confusion Matrix

\begin{tabular}{c|ccc}
\hline \multicolumn{2}{c}{} & \multicolumn{2}{c}{ Model Prediction } \\
\hline Expert Diagnosis & & Normal & Abnormal \\
& Normal & 1721 & 591 \\
\cline { 3 - 4 } & Abnormal & 218 & 797 \\
\hline
\end{tabular}

Table 4: Normalized Confusion Matrix

\begin{tabular}{l|ccc}
\hline & \multicolumn{3}{c}{ Model Prediction } \\
\hline \multirow{3}{*}{ Expert Diagnosis } & & Normal & Abnormal \\
\cline { 3 - 4 } & Normal & 0.74 & 0.26 \\
\cline { 3 - 4 } & Abnormal & 0.21 & 0.78 \\
\hline
\end{tabular}

Figures 
Table 5: A comparison to related works

\begin{tabular}{|c|c|c|c|c|c|c|c|}
\hline reference & input & $\begin{array}{l}\text { Number } \\
\text { of } \\
\text { patients }\end{array}$ & $\begin{array}{l}\text { Ground } \\
\text { truth }\end{array}$ & output & model & ACC/AUC & $\mathrm{SEN} / \mathrm{SPE}$ \\
\hline Spier et al.[4] & Stress and rest polar maps & 946 & $\begin{array}{c}\text { Expert } \\
\text { diagnosis }\end{array}$ & $\begin{array}{l}\text { Presence of obstructive } \\
\mathrm{CAD} \text { (normal vs. } \\
\text { abnormal) }\end{array}$ & Graph-CNN & $\begin{array}{c}91.2 \% / \\
\text { NA }\end{array}$ & $\begin{array}{c}86.9 \% / \\
95.9 \%\end{array}$ \\
\hline $\begin{array}{l}\text { Betancur et al. } \\
\text { [22] }\end{array}$ & Raw and TPD stress polar maps & 1638 & $\begin{array}{c}\text { Coronary } \\
\text { angiography }\end{array}$ & $\begin{array}{l}\text { Presence of obstructive } \\
\qquad \mathrm{CAD}>0.70 \\
\text { (normal vs. abnormal) }\end{array}$ & CNN & $\begin{array}{l}\text { NA / } \\
0.80 \%\end{array}$ & $\begin{array}{c}82.3 \% / \\
\text { NA }\end{array}$ \\
\hline $\begin{array}{c}\text { Betancur et al. } \\
\text { [6] }\end{array}$ & Combined Upright and supine stress TPD polar maps & 1160 & $\begin{array}{c}\text { Coronary } \\
\text { angiography }\end{array}$ & $\begin{array}{l}\text { Presence of obstructive } \\
\mathrm{CAD}>0.70 \text { and }>0.50 \text { in } \\
\mathrm{LAD} \\
\text { (normal vs. abnormal) }\end{array}$ & CNN & $\begin{array}{l}\text { NA / } \\
0.81 \%\end{array}$ & $\begin{array}{c}84.8 \% / \\
56.3 \%\end{array}$ \\
\hline $\begin{array}{l}\text { Apostolopoulos } \\
\text { et al.[10] }\end{array}$ & $\begin{array}{l}\text { A concatenation of Attenuation-corrected(AC) and } \\
\text { non-attenuation corrected(NAC) stress and rest } \\
\text { polar maps }\end{array}$ & 216 & $\begin{array}{c}\text { Coronary } \\
\text { angiography }\end{array}$ & $\begin{array}{l}\text { Presence of obstructive } \\
\qquad A D \text { (normal vs. } \\
\text { abnormal) }\end{array}$ & $\begin{array}{l}\text { Vgg-16 model using data } \\
\text { augmentation and transfer } \\
\text { learning methods }\end{array}$ & $\begin{array}{c}74.53 \% / \\
\text { NA }\end{array}$ & $\begin{array}{l}0.75 \% / \\
73.43 \%\end{array}$ \\
\hline $\begin{array}{l}\text { Apostolopoulos } \\
\text { et al.[9] }\end{array}$ & $\begin{array}{c}\text { A concatenation of (AC) and (NAC) stress and rest } \\
\text { polar maps + clinical data }\end{array}$ & 566 & $\begin{array}{c}\text { Coronary } \\
\text { angiography }\end{array}$ & $\begin{array}{l}\text { Presence of obstructive } \\
\text { CAD(normal vs. } \\
\text { abnormal) }\end{array}$ & Inceptionv3 + random forest & $\begin{array}{l}79.26 \% / \\
78.44 \%\end{array}$ & $\begin{array}{c}77.36 \% \text { I } \\
79.25 \%\end{array}$ \\
\hline $\begin{array}{c}\text { Berkaya et al. } \\
\text { [11] }\end{array}$ & $\begin{array}{l}\text { SPECT short, vertical, and horizontal long-axis } \\
\text { slices }\end{array}$ & 192 & $\begin{array}{c}\text { Expert } \\
\text { diagnosis }\end{array}$ & $\begin{array}{l}\text { Presence of abnormal } \\
\text { perfusion; (normal vs. } \\
\text { abnormal) }\end{array}$ & $\begin{array}{l}\text { Pre-trained CNN models + svm } \\
\text { classifier and a knowledge-based } \\
\text { method }\end{array}$ & $\begin{array}{l}0.94 \% \\
/ \text { NA }\end{array}$ & $0.88 \% / 1$ \\
\hline Chen et al.[13] & SPECT short, vertical and horizontal long axis slices & 979 & $\begin{array}{c}\text { Expert } \\
\text { diagnosis }\end{array}$ & $\begin{array}{l}\text { Presence of obstructive } \\
\qquad \mathrm{CAD} \text { (normal vs. } \\
\text { abnormal) }\end{array}$ & 3D CNN & $\begin{array}{c}87.64 \% / \\
\text { NA }\end{array}$ & $\begin{array}{c}81.58 \% / \\
92.16 \%\end{array}$ \\
\hline Liu et al.[14] & $\begin{array}{l}\text { circumferential count profile maps extracted from } \\
\text { SPECT images + clinical data }\end{array}$ & 37243 & $\begin{array}{c}\text { Expert } \\
\text { diagnosis }\end{array}$ & $\begin{array}{l}\text { Presence of obstructive } \\
\mathrm{CAD} \text { (normal vs. } \\
\text { abnormal) }\end{array}$ & Resnet-based CNN & $\begin{array}{c}82.7 \% / \\
0.87 \%\end{array}$ & $\begin{array}{l}74.4 \% / \\
84.9 \%\end{array}$ \\
\hline Current work & A combination of stress and rest polar maps & 3318 & $\begin{array}{c}\text { Expert } \\
\text { diagnosis }\end{array}$ & $\begin{array}{l}\text { Presence of obstructive } \\
\qquad \mathrm{CAD} \text { (normal vs. } \\
\text { abnormal) }\end{array}$ & $\begin{array}{l}\text { A 10-layer } \mathrm{CNN}+\text { data } \\
\text { augmentation }\end{array}$ & $\begin{array}{c}75.62 \% / \\
84.50 \%\end{array}$ & $\begin{array}{c}78.56 \% / \\
74.34 \%\end{array}$ \\
\hline
\end{tabular}
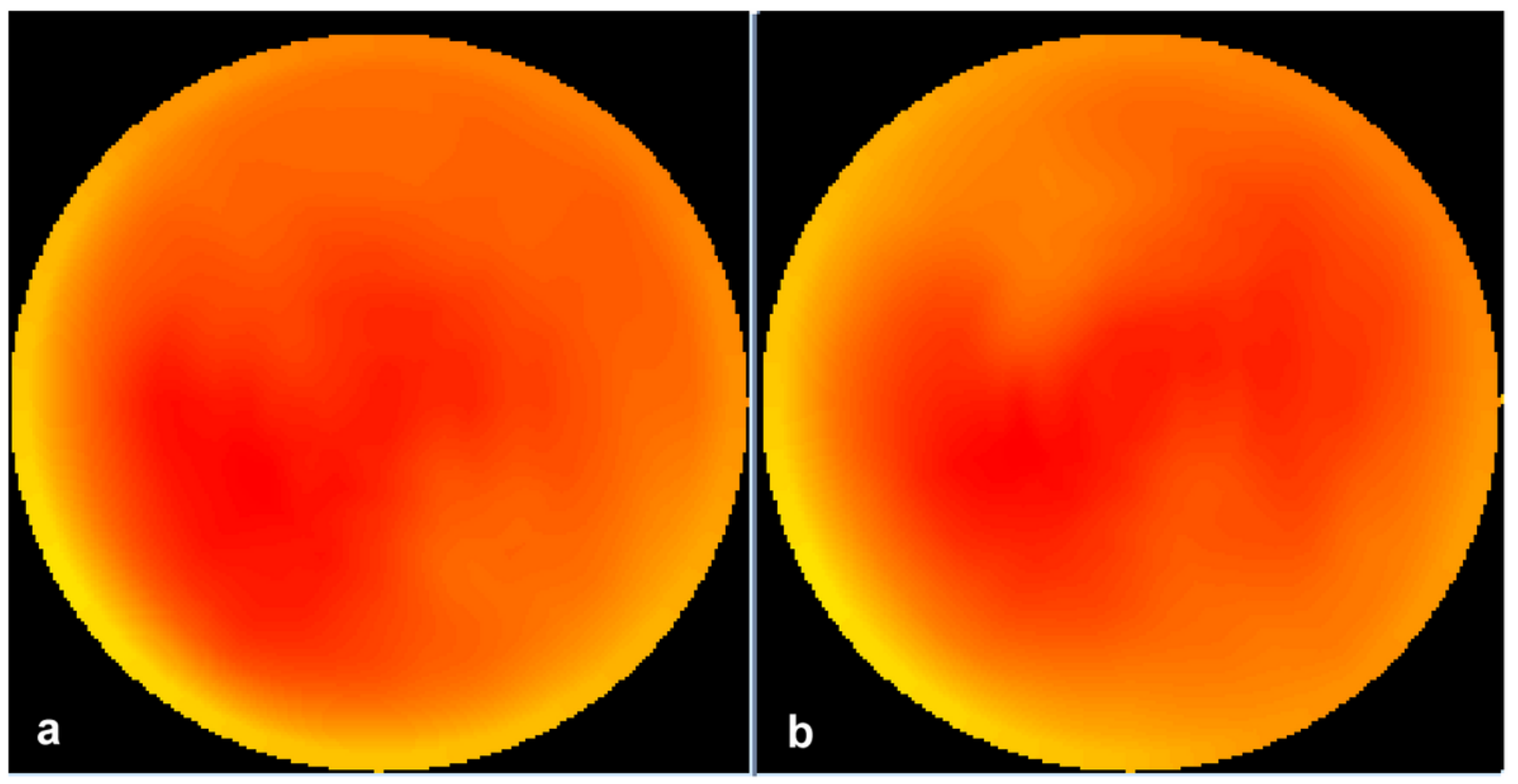

Figure 1

Normal Polar Map Images a) Under Stress and b) At Rest 

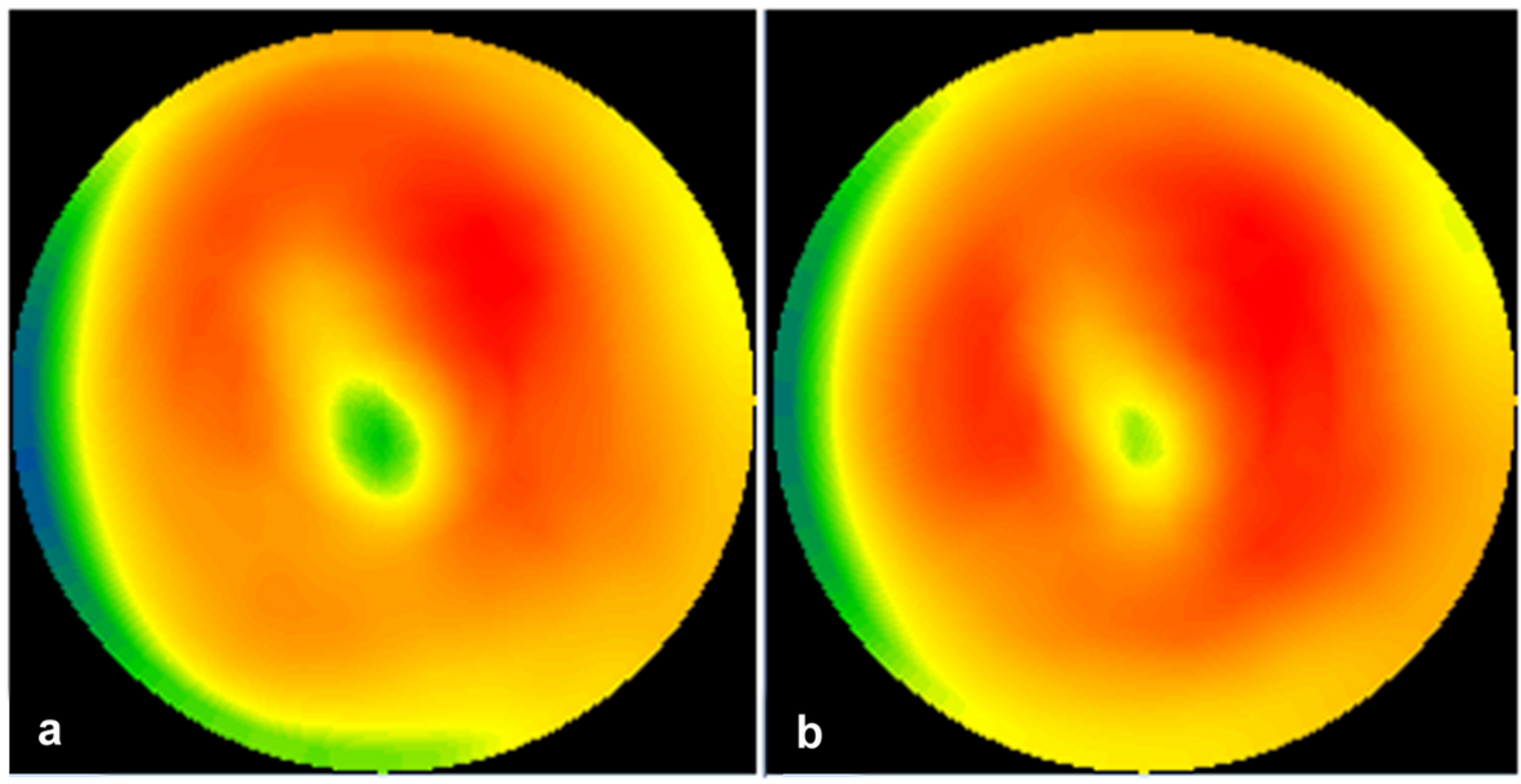

Figure 2

Abnormal Polar Map Images a) Under Stress and b) At Rest

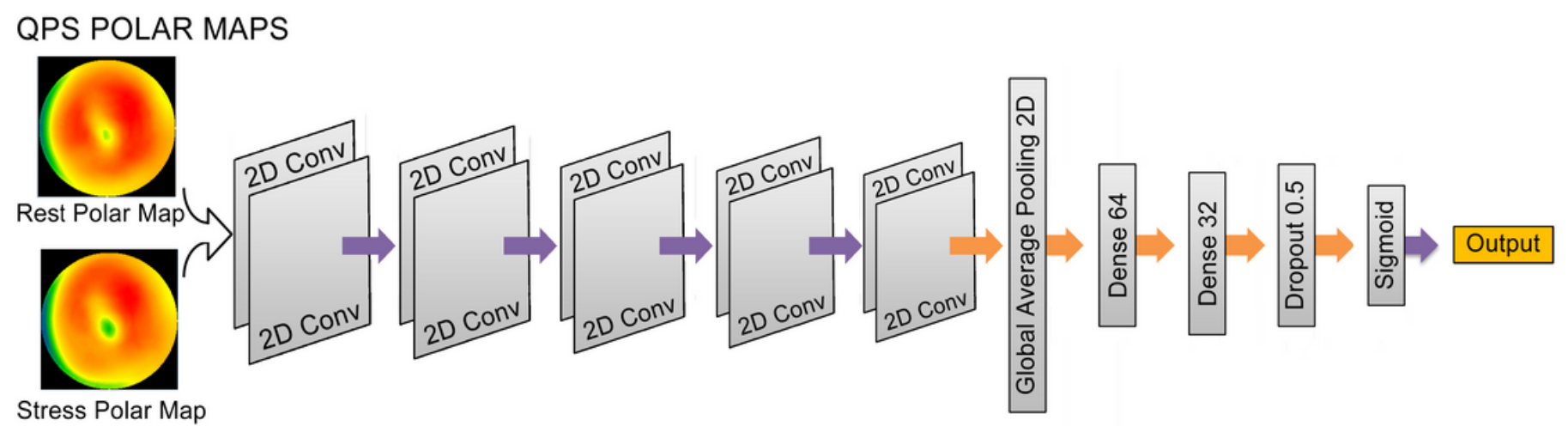

Figure 3

General Diagram of the Proposed Model 


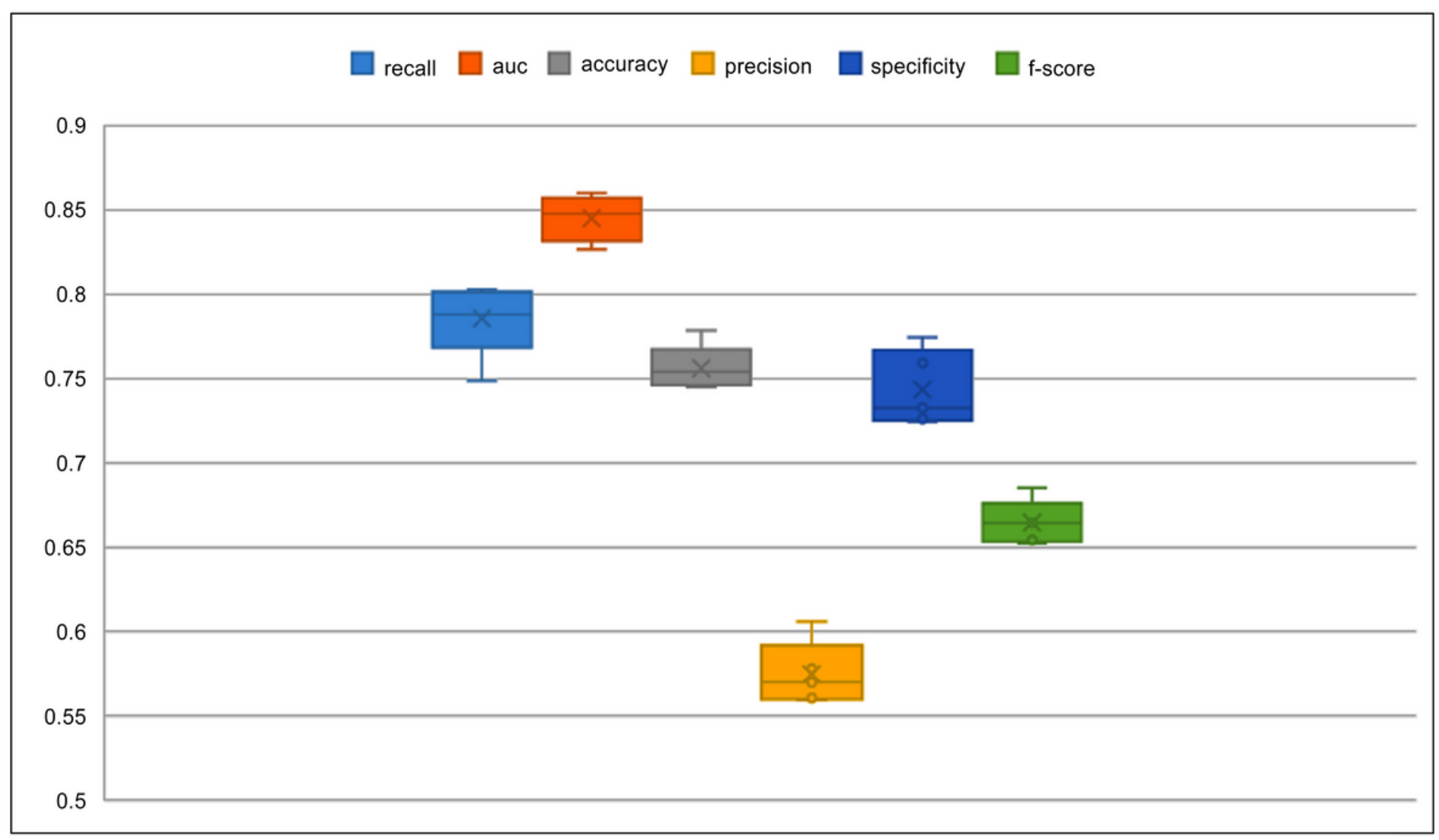

Figure 4

Distribution of Classification Metrics in Cross-Validation over 5 Folds
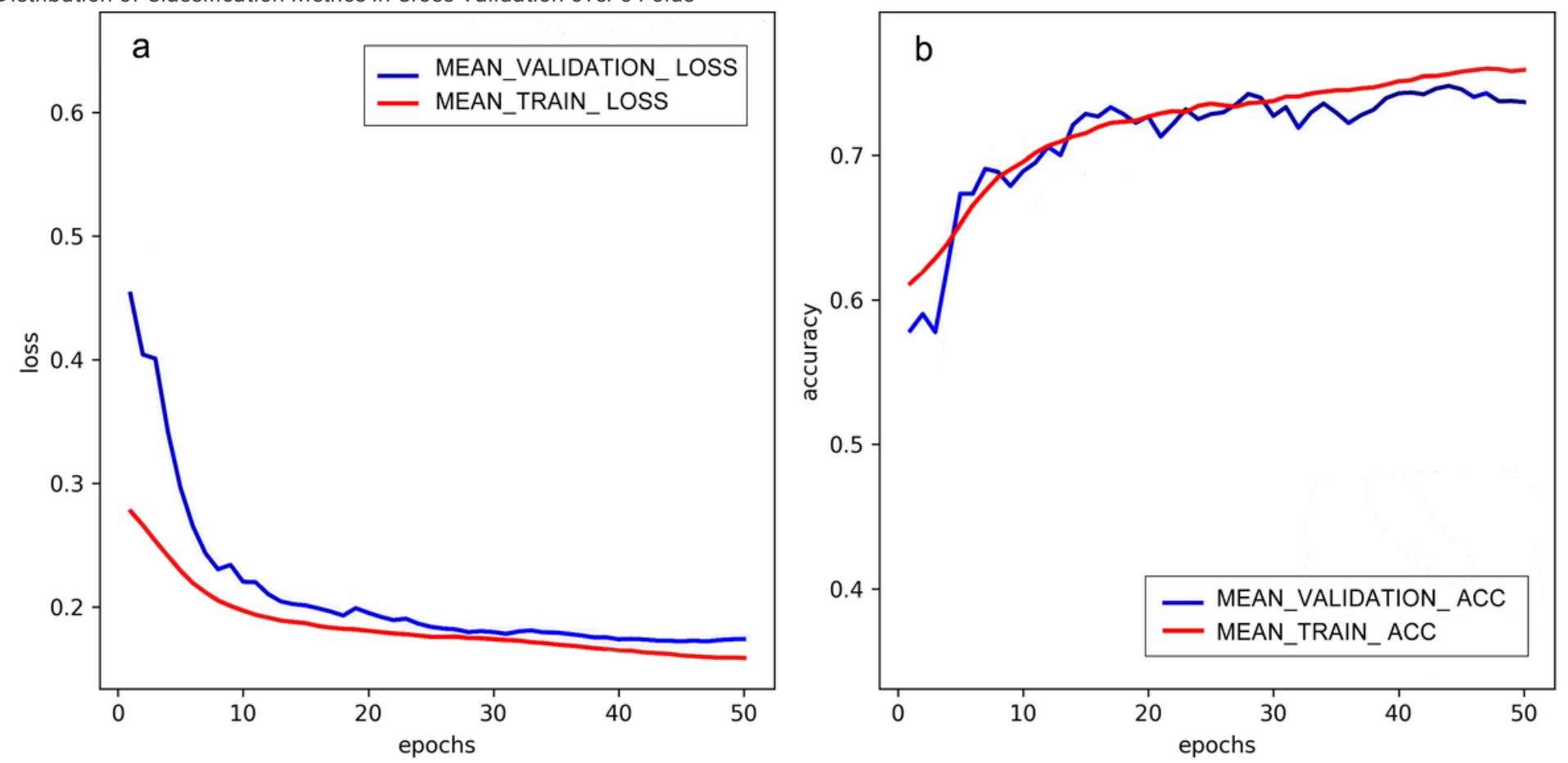

Figure 5

Average Learning Curves over 5 Folds; a) train and validation loss b) train and validation accuracy 

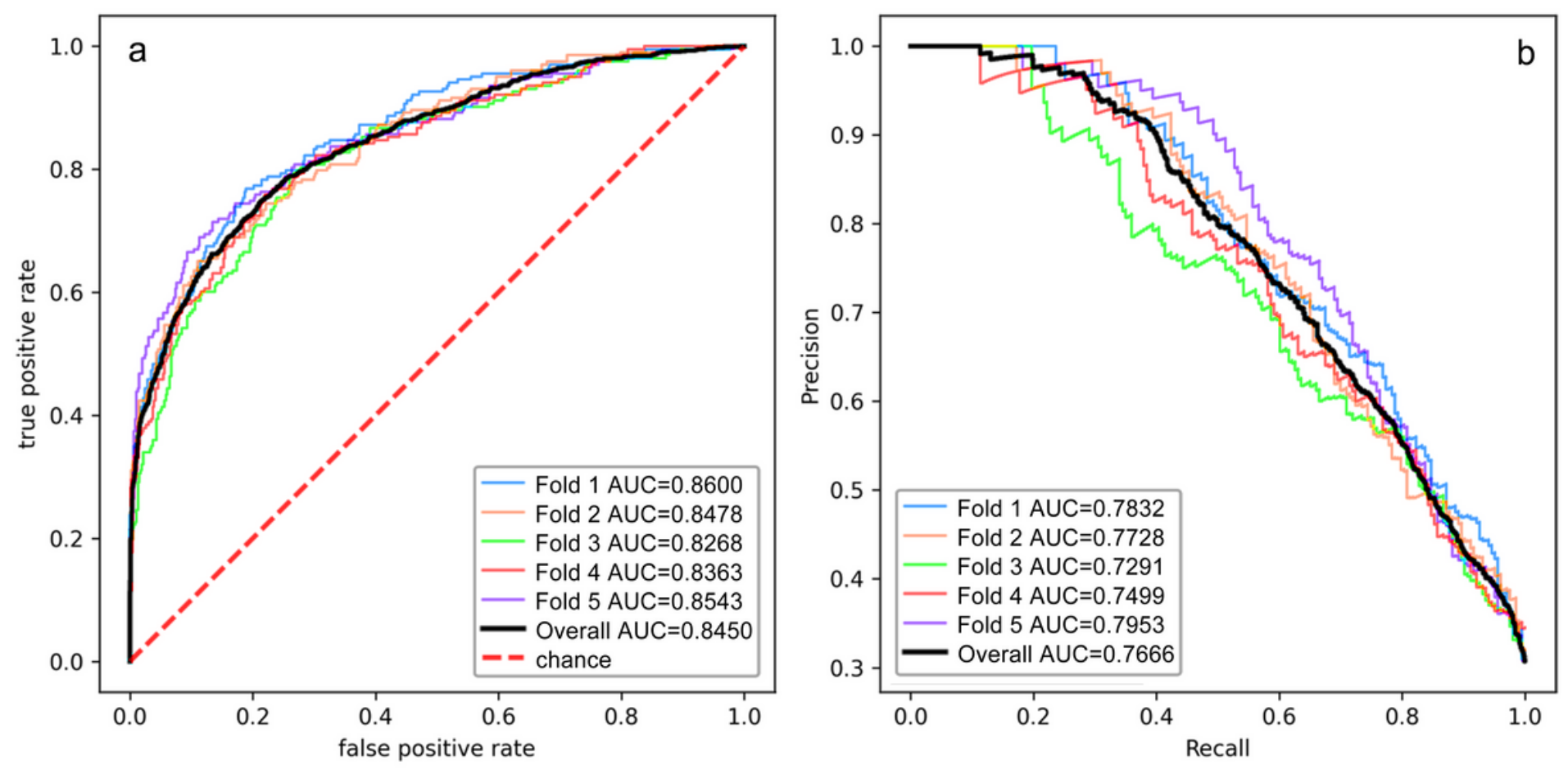

Figure 6

a) Roc and b) Precision-Recall Curves in Cross-Validation over 5 Folds

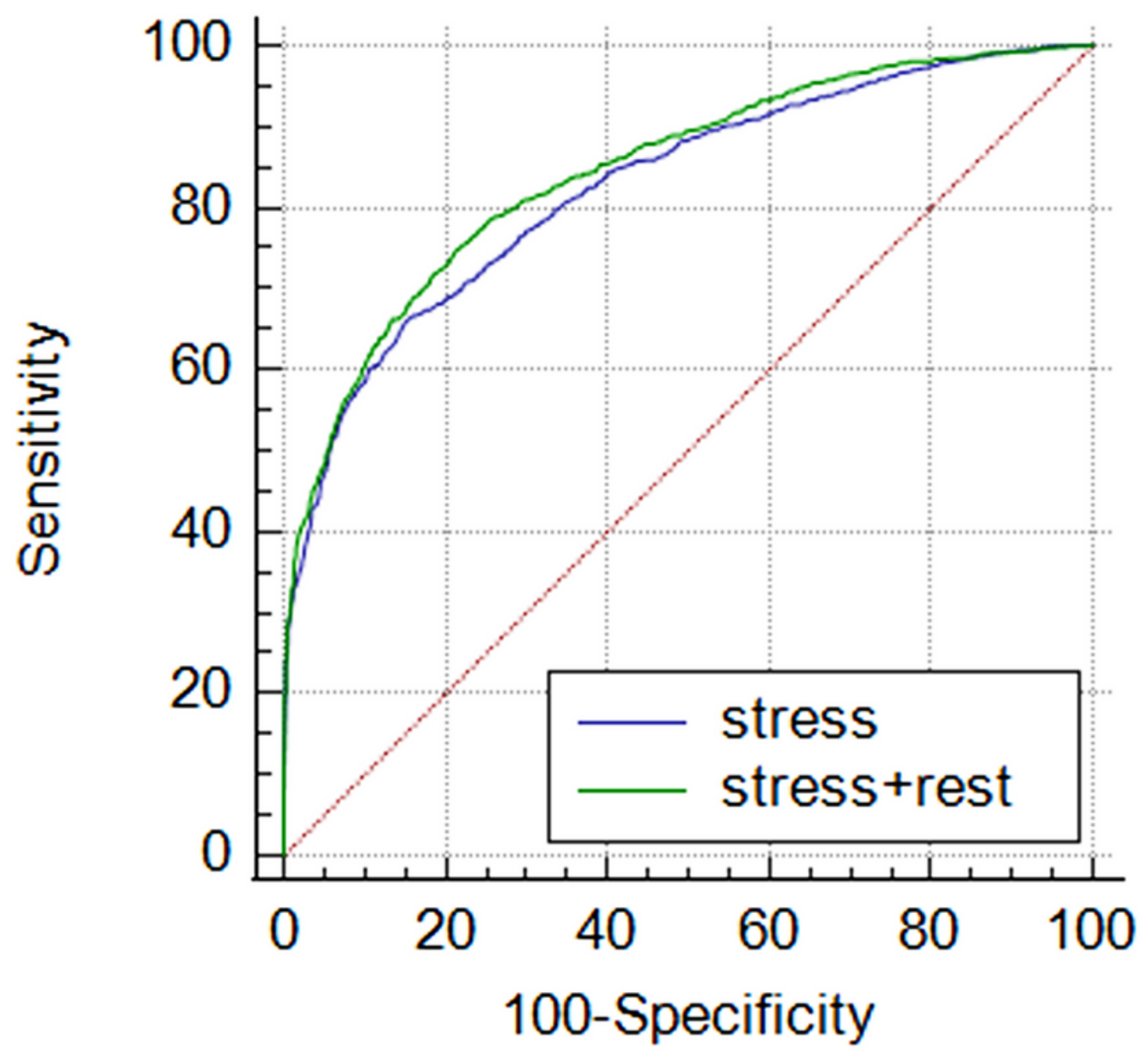


Pairwise AUC Comparison between Two DL Methods (With and Without Considering Rest Polar Maps)

Page $12 / 12$ 